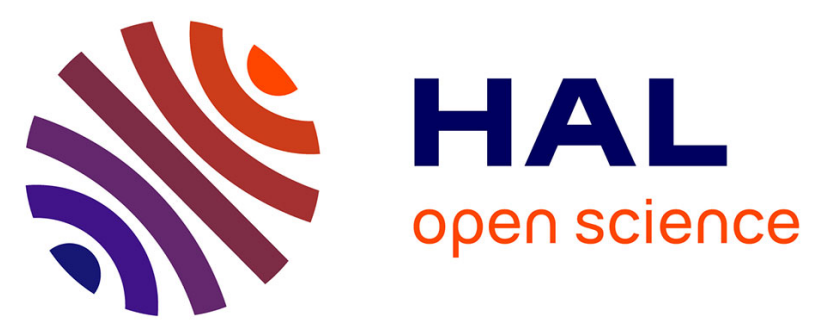

\title{
Tight Kernels for Covering and Hitting: Point Hyperplane Cover and Polynomial Point Hitting Set
}

\author{
J.-D Boissonnat, Kunal Dutta, Arijit Ghosh, Sudeshna Kolay
}

\section{To cite this version:}

J.-D Boissonnat, Kunal Dutta, Arijit Ghosh, Sudeshna Kolay. Tight Kernels for Covering and Hitting: Point Hyperplane Cover and Polynomial Point Hitting Set. LATIN 2018 - 13th Latin American Theoretical INformatics Symposium, Apr 2018, Buenos Aires, Argentina. hal-01669884

\section{HAL Id: hal-01669884 https://hal.inria.fr/hal-01669884}

Submitted on 21 Dec 2017

HAL is a multi-disciplinary open access archive for the deposit and dissemination of scientific research documents, whether they are published or not. The documents may come from teaching and research institutions in France or abroad, or from public or private research centers.
L'archive ouverte pluridisciplinaire HAL, est destinée au dépôt et à la diffusion de documents scientifiques de niveau recherche, publiés ou non, émanant des établissements d'enseignement et de recherche français ou étrangers, des laboratoires publics ou privés. 


\title{
Tight Kernels for Covering and Hitting: POINT Hyperplane Cover and Polynomial Point Hitting SeT
}

\author{
Jean-Daniel Boissonnat ${ }^{1}$, Kunal Dutta ${ }^{1}$, Arijit Ghosh $(\varangle)^{2}$, and Sudeshna \\ Kolay $^{3}$ \\ 1 Université Côte d'Azur, Inria, France \\ \{Jean-Daniel.Boissonnat| Kunal.Dutta\}@inria.fr \\ 2 Indian Statistical Institute, Kolkata, India \\ agosh@mpi-inf .mpg.de \\ 3 Eindhoven University of Technology, Netherlands \\ s.kolay@tue.nl
}

\begin{abstract}
The Point Hyperplane Cover problem in $\mathbb{R}^{d}$ takes as input a set of $n$ points in $\mathbb{R}^{d}$ and a positive integer $k$. The objective is to cover all the given points with a set of at most $k$ hyperplanes. The $D$-Polynomial Points Hitting Set ( $D$-Polynomial Points HS) problem in $\mathbb{R}^{d}$ takes as input a family $\mathcal{F}$ of $D$-degree polynomials from a vector space $\mathcal{R}$ in $\mathbb{R}^{d}$, and determines whether there is a set of at most $k$ points in $\mathbb{R}^{d}$ that hit all the polynomials in $\mathcal{F}$. For both problems, we exhibit tight kernels where $k$ is the parameter.
\end{abstract}

\section{Introduction}

A set system is a tuple $(U, \mathcal{F})$ where $U$ is a universe of $n$ elements and $\mathcal{F}$ is a family of $m$ subsets of $U$. A set system is also referred to as a hypergraph, with the elements in the universe $U$ named as vertices and the subsets in $\mathcal{F}$ named as hyperedges. A hyperedge is said to cover a vertex if the vertex belongs to the hyperedge. Similarly a subfamily $\mathcal{F}^{\prime}$ of hyperedges is said to cover a subset $V$ of vertices if for each vertex $v \in V$ there is a hyperedge $h \in \mathcal{F}^{\prime}$ such that $h$ covers $v$. A vertex is said to hit a hyperedge if the vertex belongs to the hyperedge, and a subset $V$ of vertices is said to hit a subfamily $\mathcal{F}^{\prime}$ of hyperedges if for each hyperedge $h \in \mathcal{F}^{\prime}$ there is a vertex $v \in V$ that belongs to $h$.

The Set Cover and Hitting Set problems are two of the most well-studied problems in computer science. For the SET Cover problem, the input is a set system $(U, \mathcal{F})$ and a positive integer $k$. The objective is to determine whether there is a subfamily $\mathcal{F}^{\prime} \subseteq \mathcal{F}$ with at most $k$ subsets, such that $\mathcal{F}^{\prime}$ covers all the elements in $U$. Such a family $\mathcal{F}^{\prime}$ is referred to as a solution family or a covering family. The Hitting Set problem can be thought of as a dual problem. Here, the input is the same as in SET Cover. However, now the objective is to determine whether there is a subset $S \subseteq U$ of size at most $k$, such that for each hyperedge $h \in \mathcal{F}, h \cap S \neq \emptyset$. Such a set $S$ is referred to as a solution set. 
These problems are part of the original 21 NP-complete problems posed by Richard Karp [15]. However, the numerous applications for these problems inspired researchers to design algorithms to find solutions with reasonable efficiency, for different measures of efficiency. For SET Cover, the best approximation factor is $\mathcal{O}(\log n)$ [20]. It was shown in [9] that $\log n$ is the best possible approximation factor unless P $=$ NP. Since Hitting SET is just a reformulation of the SET Cover problem, the same approximation factors hold. The $d$-Hitting SET problem, where the size of each subset in $\mathcal{F}$ is exactly $d$, is known to be APX-hard [2], consequent to results obtained for the special case of the VERTEX COVER problem where $d=2$.

Set Cover and Hitting Set have been studied in parameterized complexity. In parameterized complexity, we say that a problem is fixed parameter tractable (FPT) with respect to a parameter $k$, if there is an algorithm that takes an instance of size $n$ of the problem, and solves the problem in $f(k) . n^{\mathcal{O}(1)}$ time, where $f$ is a computable function. For a brief introduction to parameterized complexity please refer to the Preliminaries. For further details please refer to [7, 11,12 . The $d$-Hitting SET problem, parameterized by the solution size $k$, is known to be FPT, with a tight $\mathcal{O}\left(k^{d}\right)$-sized kernel [8] under standard complexity theoretic assumptions. In this paper, unless otherwise mentioned, all variants of Hitting Set and Set Cover are parameterized by $k$. The Set Cover and the general Hitting SeT problems are W[2]-hard, and are not expected to be FPT.

Interestingly, the instances of many real world applications of these two notoriously hard problems have inherent structure in them. With the hope of designing efficient algorithms for such instances by exploiting their structural information, numerous variants of SET Cover and Hitting Set have been studied. A very natural extension in this field of study is to assume geometric structure on the instances. In recent years, there has been a lot of attention to study geometric variants of both the problems.

The Point Line Cover is an example of a geometric variant of Set Cover, where the universe is a set of points in $\mathbb{R}^{2}$ and the hyperedges are the maximal sets of collinear points in the input. POINT Line Cover is known to be FPT [17]. Kratsch et al. showed in [16] that the problem has a tight polynomial kernel with $\mathcal{O}\left(k^{2}\right)$ points. In [1], several generalizations of the Point Line Cover problem were studied - a universe is a set of points in a Euclidean space and the family of hyperedges are geometric structures like hyperplanes, spheres, curves, etc. Geometric variants of Set Cover have been studied in $[3,4]$.

The results in [16] also imply parameterized results for Line Point Hitting SET, where the universe is a set of lines in $\mathbb{R}^{2}$ and the objective is to find at most $k$ points in $\mathbb{R}^{2}$ to hit the universe of lines. This problem is FPT and has a tight kernel with $\mathcal{O}\left(k^{2}\right)$ lines. Other geometric variants of the Hitting Set problem has been studied in parameterized complexity $[10,13,14]$. Bringmann et al. [6] studied the problem for set systems with bounded VC dimensions. They showed that there are set systems with $\mathrm{VC}$ dimension as low as 2, where both the Hitting Set, and consequently the Set Cover problem are W[1]-hard. This 
gives an interesting dichotomy, since they also show that when the $\mathrm{VC}$ dimension of the set system is 1, then the Hitting SET problem is in P.

In this paper, we consider two parameterized variants.

POINT HYPERPlane COVER IN $\mathbb{R}^{d} \quad$ Parameter: $k$
Input: A set $\mathcal{P}$ of $n$ points in $\mathbb{R}^{d}$, a positive integer $k$.
Question: Is there a family of at most $k$ hyperplanes in $\mathbb{R}^{d}$ that cover all the
points in $\mathcal{P}$ ?

We also study the Projective Point Hyperplane Cover problem, where the family of hyperplanes allowed to cover the input set of points must pass through the origin in $\mathbb{R}^{d}$, and we are not allowed to include the origin in the input set of points. Note that this problem is equivalent to that of covering points on a sphere with the great circles(radius is equal to the radius of the sphere) of the sphere, which has many applications in computational geometry.

$D$-Polynomial Point $\mathrm{HS}^{a}$ IN $\mathbb{R}^{d}$

Parameter: $k$

Input: A set $\mathcal{F}$ of $n D$-degree polynomials from a specified vector space $\mathcal{R}$ of $D$-degree polynomials in $\mathbb{R}^{d}$, a positive integer $k$.

Question: Is there a set $S$ of at most $k$ points in $\mathbb{R}^{d}$ such that for each polynomial $f \in \mathcal{F}$, there is a point $p \in S$ with $f(p)=0$ ?

${ }^{a} \mathrm{HS}$ is a shorthand for hitting set.

Please refer to the Preliminaries for the definition of $D$-degree polynomials. Both problems are NP-hard, because of the NP-hardness of POINT Line Cover [19]. Parameterized by $k$, we study the parameterized complexity of these problems.

Our results. Extending the results of Kratsch et al. [16], we show that POINT Hyperplane Cover and Projective Point Hyperplane Cover in $\mathbb{R}^{d}$ have tight polynomial kernels with $\mathcal{O}\left(k^{d}\right)$ and $\mathcal{O}\left(k^{d-1}\right)$ points, respectively. These results are presented in Section 3. The highlight of this proof is, given any positive integer $n$, the construction of a set $\mathcal{P}$ of $n$ points in $\mathbb{R}^{d}$ in general position such that the family of hyperplanes, defined by any $d$ points from $\mathcal{P}$, do not have too many hyperplanes intersecting at a point outside $\mathcal{P}$. This is crucial for a many-one reduction from $d$-HitTing SET, that results in lower bounds on the size as well as the number of points in a kernel under complexity theoretic assumptions. The construction is similar to that in [16] in spirit, but requires more geometric insight since we are working in higher dimensions now.

Note that the results of Point Hyperplane Cover also imply that the dual problem, Hyperplane PoINT HS in $\mathbb{R}^{d}$, also has a tight kernel with $\Omega\left(k^{d}\right)$ hyperplanes. Similarly, we can show that Projective Hyperplane Point Cover has a tight kernel with $\Omega\left(k^{d-1}\right)$ hyperplanes.

Our main contribution is to show tight polynomial bounds for kernel sizes for $D$-Polynomial Point HS in $\mathbb{R}^{d}$, for a large family of vector spaces $\mathcal{R}$ of $D$-degree polynomials. For more details on the characterization of $\mathcal{R}$, please see the Preliminaries. The vector space of hyperplanes, spheres and ellipses are among 
natural vector spaces of polynomials that are covered by this characterization. Therefore, our techniques provide a general framework for proving tight kernels for covering problems, as one can get a tight bound for many families directly. This result is given in Section 4.

Our proof strategy is to use the Veronese mapping [18] to transform the space of points and polynomials to a higher dimensional space, where the polynomials transform into hyperplanes and point-polynomial incidences are preserved. The upper bound on the kernel size comes directly from the Veronese mapping. For the lower bound, we show that points in general position with respect to polynomials transform to an equal-sized set of points in general position with respect to hyperplanes in the image space. Using this fact, we construct hard instances of $D$-Polynomial Point HS. In [5], the Veronese mapping was used to give upper bounds on kernel sizes. In this paper, we also utilize the map to exhibit kernel lower bounds.

\section{Preliminaries}

Multivariate Polynomials. Given a set $\left\{X_{1}, X_{2}, \ldots, X_{d}\right\}$ of variables a real multivariate polynomial on these variables is of the form $P\left(X_{1}, \ldots, X_{d}\right)=$ $\sum_{i_{1}, i_{2}, \ldots, i_{d}} a_{i_{1} i_{2} \ldots i_{d}} \prod_{j \in[d]} X_{j}^{i_{j}}$ where $[d]=\{1, \ldots, d\}$ and $a_{i_{1} i_{2} \ldots i_{d}} \in \mathbb{R}$. The set of all real multivariate polynomials in the variables $X_{1}, \ldots, X_{d}$ will be denoted by $\mathbb{R}\left[X_{1}, X_{2}, \ldots, X_{d}\right]$. The degree of such a polynomial $P\left(X_{1}, \ldots, X_{d}\right)$ is defined as $\operatorname{deg}(P):=\max \left\{i_{1}+i_{2}+\ldots+i_{d} \mid a_{i_{1} i_{2} \ldots i_{d}} \neq 0\right\}$. A polynomial is said to be a $D$-degree polynomial if its degree is $D$.

In this paper, we are interested in the set/subsets of polynomials whose degree is bounded by $D$, for some $D \in \mathbb{N}$. In this context we define $\operatorname{Poly}_{D}\left[X_{1}, \ldots, X_{d}\right]:=$ $\left\{f\left(X_{1}, \ldots, X_{d}\right) \in \mathbb{R}\left[X_{1}, \ldots, X_{d}\right] \mid \operatorname{deg}(f) \leq D\right\}$. Observe that the vector space Poly $_{D}\left[X_{1}, \ldots, X_{d}\right]$ over $\mathbb{R}$ has the monomials $\left\{X_{1}^{i_{1}} \ldots X_{d}^{i_{d}} \mid 0 \leq \sum_{j=1}^{d} i_{j} \leq D\right\}$ as a basis and $\left|\left\{X_{1}^{i_{1}} \ldots X_{d}^{i_{d}} \mid 0 \leq \sum_{j=1}^{d} i_{j} \leq D\right\}\right|=\left(\begin{array}{c}D+d \\ D\end{array}\right)$. For ease of notation, we define the vector $X=\left(X_{1}, \ldots, X_{d}\right)$.

Given a polynomial $f$ and a point $p$, the point hits the polynomial if $f(p)=0$. In the same situation, the polynomial is said to cover the point.

General position in Geometry. An $i$-flat in $\mathbb{R}^{d}$ is the affine hull of $i+1$ affinely independent points. The dimension of a (possibly infinite) set of points $\mathcal{P}$, denoted as $\operatorname{dim}(\mathcal{P})$, is the minimum $i$ such that the entire set $\mathcal{P}$ is contained in an $i$-flat of $\mathbb{R}^{d}[17]$. We use the term hyperplanes interchangeably for $(d-1)$-flats. A set $\mathcal{P}$ of points in $\mathbb{R}^{d}$ is said to be in general position with respect to hyperplanes, if for each $i$-flat, $i \leq d-1$, in $\mathbb{R}^{d}$ there are at most $i+1$ points from $\mathcal{P}$ lying on the $i$-flat.

Consider, for $i \leq d-1$, a family $\mathcal{F}$ of $i$-flats such that there is a point $p$ that belongs to all the $i$-flats in $\mathcal{F}$. Then a set $\mathcal{P} \in \mathbb{R}^{d} \backslash\{p\}$ of points is said to be in general position with respect to $\mathcal{F}$ if each $i$-flat contains at most $i$ points from $\mathcal{P}$ lying on the $i$-flat. This is called general position in projective geometry. 
Similarly, we can define the notion of general position (resp. projective general position) with respect to multivariate polynomials. Let $\mathcal{R}$ be a vector space of Poly $_{D}[X]$, defined by a basis $\left\{f_{1}(X), \ldots, f_{b}(X), 1\right\}$ (resp. by a basis $\left\{f_{1}(X), \ldots, f_{b}(X)\right\}$ with $\left.\operatorname{deg}\left(f_{i}\right)>0\right)$. A subset of points is said to be in general position (resp. projective general position) with respect to the vector space $\mathcal{R}$ of polynomials if no more than $b$ points (resp. $b-1$ points) from the subset satisfy any equation of the form $f(X):=\sum_{i=1}^{b} \lambda_{i} f_{i}(X)+\lambda_{b+1}=0$ $\left(f(X):=\sum_{i=1}^{b} \lambda_{i} f_{i}(X)=0\right)$, where all the $\lambda_{j} \in \mathbb{R}$ and not all the $\lambda_{j}$ 's can be zero simultaneously.

Definition 1. Given a rational number $\alpha>0$, a vector space $\mathcal{R}$ of polynomials in $\mathbb{R}^{d}$ is said to be $\alpha$-good if for any positive integers $b, m$ the following conditions hold:

1. In $\mathcal{O}(1)$ time we can compute a set of b points in $\mathbb{R}^{d}$ such that the set is in general position with respect to $\mathcal{R}$.

2. Given a d-dimensional $m \times \cdots \times m$ grid in $\mathbb{R}^{d}$, each polynomial in $\mathcal{R}$ contains at most $m^{d-\alpha}$ vertices of the grid.

Hyperplanes, spheres, ellipses and many other natural vector spaces of polynomials can be described as $\alpha$-good vector spaces.

Veronese mapping. In this paper, one of our strategies for generalizing our results is to convert $D$-Polynomial Point HS in $\mathbb{R}^{d}$ to Hyperplane Point HS in $\mathbb{R}^{b}$ by using a variant of Veronese mapping [18] from $\mathbb{R}^{d} \rightarrow \mathbb{R}^{b}$. The Veronese mapping of a vector space $\mathcal{R}$ of $D$-degree polynomials, with a basis $\left\{f_{1}(X), \ldots, f_{b}(X), 1\right\}$ (also with a basis $\left\{f_{1}(X), \ldots, f_{b}(X)\right\}$ where $\left.\operatorname{deg}\left(f_{i}\right)>0\right)$, is as follows- $\Phi_{\mathcal{R}}: \mathbb{R}^{d} \rightarrow \mathbb{R}^{b}$, where $\Phi_{\mathcal{R}}(X)=\left(f_{1}(X), \ldots, f_{b}(X)\right)$ where $X=\left(X_{1}, \ldots, X_{d}\right)$. Observe that if $p=\left(p_{1}, \ldots, p_{d}\right)$ satisfies the equation $f(X):=\sum_{i=1}^{b} \lambda_{i} f_{i}(X)+\lambda_{b+1}=0$ (resp. the equation $\left.f(X):=\sum_{i=1}^{b} \lambda_{i} f_{i}(X)=0\right)$ then $\Phi_{\mathcal{R}}(p)$ will also satisfy the linear equation $\sum_{j=1}^{b} \lambda_{j} Z_{j}+\lambda_{b+1}=0$ (or the equation $\sum_{j=1}^{b} \lambda_{j} Z_{j}=0$ ), on the variable vector $Z=\left(Z_{1}, \ldots, Z_{b}\right)$. In other words, for any set of points $\mathcal{P}$ in $\mathbb{R}^{d}$ and $\mathcal{F}$, the incidences between $\mathcal{P}$ and $\mathcal{R}$ and incidences between $\Phi_{\mathcal{R}}(\mathcal{P})$ and hyperplanes in $\mathbb{R}^{b}$ (or the hyperplanes passing through the origin in $\mathbb{R}^{b}$ ) are preserved under the mapping $\Phi_{\mathcal{R}}$. Also, observe that there is a bijection between polynomials in $\mathcal{R}$ and hyperplanes in $\mathbb{R}^{b}$ (resp. hyperplanes passing through the origin in $\left.\mathbb{R}^{b}\right)$. This transformation from polynomials to hyperplanes is also referred to as linearization.

Parameterized Complexity. The instance of a parameterized problem or language is a pair containing the actual problem instance of size $n$ and a positive integer called a parameter, usually represented as $k$. The problem is said to be in FPT if there exists an algorithm that solves the problem in $f(k) \cdot n^{\mathcal{O}(1)}$ time, where $f$ is a computable function. The problem is said to admit a $g(k)$-sized kernel, if there exists a polynomial time algorithm that converts the actual instance to a reduced instance of size $g(k)$, while preserving the answer. When $g$ is a 
polynomial function, then the problem is said to admit a polynomial kernel. A reduction rule is a polynomial time procedure that changes a given instance $I_{1}$ of a problem $\Pi$ to another instance $I_{2}$ of the same problem $\Pi$. We say that the reduction rule is safe when $I_{1}$ is a Yes instance of $\Pi$ if and only if $I_{2}$ is a YeS instance. Readers are requested to refer to [7] for more details on Parameterized Complexity.

Lower bounds in Parameterized Algorithms. There are several methods of showing lower bounds in parameterized complexity under standard assumptions in complexity theory. In this paper we require a lower bound technique given by Dell and Melkebeek [8]. This technique links kernelization to oracle protocols.

Definition 2. Given a language $L$, an oracle communication protocol for $L$ is a two-player communication protocol. The first player gets an input $x$ and can only execute computations taking time polynomial in $|x|$. The second player is computationally unbounded, but does not know $x$. At the end of the protocol, the first player has to decide correctly whether $x \in L$. The cost of the protocol is the number of bits of communication from the first player to the second player.

Proposition 3. [8] Let $d \geq 2$ be an integer, and $\epsilon$ be a positive real number. If co-NP $\nsubseteq \mathrm{NP} /$ poly, then there is no protocol of cost $\mathcal{O}\left(n^{d-\epsilon}\right)$ to decide whether a d-uniform hypergraph on $n$ vertices has a d-hitting set of at most $k$ vertices, even when the first player is co-nondeterministic.

As noted in [8], this implies that for any $d \geq 2$ and any positive real number $\epsilon$,

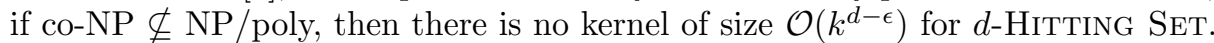
In general, a lower bound for oracle communication protocols for a parameterized language $L$ gives a lower bound for kernelization for $L$.

Kernels: size vs number of elements. In the literature, a lower bound on the kernel implies a lower bound on the size in bits of the kernel, but not necessarily on the number of input elements in the kernel. Kratsch et al. [16] were one of the first to study lower bounds in terms of the number of input elements in the kernel. They used the results of Dell and Melkebeek [8] along with results in two dimensional geometry to build a new technique to show lower bounds for the number of input elements in a kernel for a problem. In this paper, we have adhered to the general convention by saying that a kernel has a lower bound on its size if it has a lower bound on its representation in bits, while explicitly mentioning the cases where the kernel has a lower bound on the number of input elements.

\section{Kernelization Lower bound for Point HyPERPLANE COVER}

In this Section, we show that Point Hyperplane Cover in $\mathbb{R}^{d}$ has a tight kernel of size $\Theta\left(k^{d}\right)$. The results in [17] imply that Point Hyperplane Cover 
in $\mathbb{R}^{d}$ has a kernel of size $\mathcal{O}\left(k^{d}\right)$. We show that the problem cannot have a kernel of size $\mathcal{O}\left(k^{d-\epsilon}\right)$ if co-NP $\nsubseteq \mathrm{NP} /$ poly. We show this by the standard technique of polynomial parameter transformation. For a fixed $d$, we reduce the $d$-HitTing Set problem to the problem of Point Hyperplane Cover in $\mathbb{R}^{d}$. We first state the folklore equivalence between Point Hyperplane Cover in $\mathbb{R}^{d}$ and Hyperplane Point HS in $\mathbb{R}^{d}$.

Lemma 4. Point Hyperplane Cover in $\mathbb{R}^{d}$ and Hyperplane Point HS in $\mathbb{R}^{d}$ are equivalent problems

From now on, we will be showing lower bounds for HyPERPlane POINT HS. The proof strategy is the same as that in [16]. For this, we construct for each positive integer $n$ and each $d$, a set of $n$ points in $\mathbb{R}^{d}$ with some special properties. This construction is more involved than in the case of Point Line Cover.

Lemma 5. For every $n \in \mathbb{Z}^{+}$, there is a poly $(n)$ time algorithm to construct a set $\mathcal{P}$ of $n$ points in $\mathbb{R}^{d}$ that have the following properties:

(1) The points are in general position.

(2) Let $\mathcal{H}$ be the family of hyperplanes defined by all sets of d points from $\mathcal{P}$. The hyperplanes in the family $\mathcal{H}$ are in general position, i.e., given $r$ hyperplanes $H_{1}, \ldots, H_{r}$ in $\mathcal{H}$ with $r \leq d$ the dimension of the affine space $\cap_{i=1}^{r} H_{r}$ is $d-r$.

(3) For any point $p$ in $\mathbb{R}^{d} \backslash \mathcal{P}$, there are at most $d$ hyperplanes in $\mathcal{H}$ that contain $p$.

Proof. The set $\mathcal{P}$ is built inductively. When $n=d$, it is the base case and the construction follows trivially by taking any $n$ points in general position. There is exactly one hyperplane that is defined by this set of $d$ points, therefore all required conditions are met. Now, assume that for $d \leq t<n$, we have constructed a point set $\mathcal{P}_{t}$ that satisfies the above conditions. As in [16], our goal will be to extend the point set $\mathcal{P}_{t}$ by one point. We will show that points forbidden to be added to the set $\mathcal{P}_{t}$ will lie on a bounded number of hyperplanes and we will call these hyperplanes forbidden hyperplanes. Observe that the number of forbidden hyperplanes arising due to condition (1) is $O\left(t^{d}\right)$. A forbidden hyperplane due to condition (2) is defined by the intersection space of a set of at most $d$ hyperplanes and a set of at most $d-1$ points from $\mathcal{P}_{t}$. Therefore, the bound on the number of forbidden hyperplanes arising from condition (2) is $O\left(t^{d^{2}+d-1}\right)$.

Unlike the case when $d=2$, it is harder to bound the number of forbidden hyperplanes due to condition (3). Let $q \in \mathbb{R}^{d}$ be a point where the point set $\mathcal{P}^{\prime}=\mathcal{P}_{t} \cup\{q\}$ satisfies conditions (1) and (2), but not condition (3). We will call such a point $q$ a forbidden point. Let $\mathcal{H}^{\prime}$ be the family of hyperplanes defined by each set of $d$ points from $\mathcal{P}^{\prime}$. Let $H_{1}, \ldots, H_{d+1}$ be a set of $d+1$ hyperplanes in $\mathcal{H}^{\prime}$ such that they intersect at point $s$ with $s \in \mathbb{R}^{d} \backslash \mathcal{P}^{\prime}$. Observe that since the point set $\mathcal{P}_{t}$ satisfied all the three conditions, $q$ will lie on at least 1 hyperplane from the family $\left\{H_{1}, \ldots, H_{d+1}\right\}$. Suppose $q$ was contained in at least $d$ hyperplanes from the family, then $q=s$ as $\mathcal{P}^{\prime}$ satisfies condition (2). Therefore, it must 
be the case that $q$ lies in at least 1 and at most $d-1$ hyperplanes from the family $\left\{H_{1}, \ldots, H_{d+1}\right\}$. Without loss of generality, assume that $q$ lies on the hyperplanes $\left\{H_{1}, \ldots, H_{r}\right\}$. Let $A_{r-1}$ denote the $(r-1)$ dimensional affine plane $\cap_{i=r+1}^{d+1} H_{i}$. For $j \in[r]$, let the hyperplanes $H_{j}$ be generated by the set $\left\{q, p_{1}^{j}, \ldots, p_{d-1}^{j}\right\} \subset \mathcal{P}^{\prime}$. The point $s$ also belongs to $A_{r-1}$. Since we are interested in understanding where the forbidden point $q$ can lie, we try to understand the inverse problem where $A_{r-1}, s$, and points $p_{\ell}^{j}$ (for all $\ell \in[d-1]$ ) are fixed and $q$ is the variable point such that $\cap_{i=1}^{d+1} H_{i}=s \in \mathbb{R}^{d} \backslash \mathcal{P}^{\prime}$. Using elementary Euclidean geometry, we get that at least $d-r+1$ coordinates of $s$ are fixed when $A_{r-1}$ gets fixed. We know that $q$ lies on a $d-r+1$ dimensional affine plane passing through $s$. Since $d-r+1$ coordinates of $s$ are fixed by $A_{r-1}$, the slope of the affine plane depends only on $A_{r-1}$ and the points $p_{\ell}^{j}, j \in[r]$ and $\ell \in[d-1]$. This implies that as we vary $s$ on $A_{r-1}$ we will span a hyperplane which only depends on $A_{r-1}$ and the points $p_{\ell}^{j}, j \in[r]$ and $\ell \in[d-1]$. Therefore, once the hyperplanes $H_{r+1}$ till $H_{d+1}$ and the point set $\left\{p_{t}^{j} \mid j \in[r], \ell \in[d-1]\right\}$ are fixed, the point $q$ will lie on a unique hyperplane. This implies that the number of forbidden hyperplanes due to condition (3) is bounded by $O\left(t^{d^{2}+d-1}\right)$.

As we have an upper bound on the number of forbidden hyperplanes, we can now use the trick of Kratsch et al. to generate points satisfying conditions (1) to (3) [16, Lemma 2.4]. In our case, we take a $d$-dimensional $m \times \cdots \times m$ grid with $m=n^{d^{2}+d}$. Observe that the number of points from this $d$-dimensional grid that can lie on any hyperplane is bounded by $m^{d-1}$.

Finally, we are ready to prove the following Theorem.

Theorem 6. HyPERPLANE POINT HS in $\mathbb{R}^{d}$ cannot have a kernel of size $\mathcal{O}\left(k^{d-\epsilon}\right)$ if co-NP $\nsubseteq \mathrm{NP} /$ poly.

Proof. We give a reduction from $d$-Hitting SET. Let $(U, \mathcal{F}, k)$ be an instance of $d$-Hitting SEt. First we reduce this instance to the following instance $\left(U^{\prime}, \mathcal{F}^{\prime}, d k\right)$ where:

1. For each $v \in U$ we make $d$ copies $\left\{v^{1}, v^{2}, \ldots, v^{d}\right\}$. We also refer to the set $\left\{v^{1}, v^{2}, \ldots, v^{d}\right\}$ as the row of $v$.

2. $U^{\prime}=U_{1} \uplus U_{2} \uplus \ldots \uplus U_{d}$ such that for each $i \in\{1, \ldots, d\} U_{i}=\left\{v^{i} \mid v \in U\right\}$.

3. $\mathcal{F} \subset \mathcal{F}^{\prime}$

4. Assume that there is an arbitrary ordering on the vertices of $U=\left\{v_{1}, v_{2}, \ldots, v_{n}\right\}$. For each $f=\left\{v_{j_{1}}, v_{j_{2}}, \ldots, v_{j_{d}}\right\} \in \mathcal{F}$, and for each $i_{1}, i_{2}, \ldots, i_{d} \in\{1, \ldots, d\}$, we create a subset $f_{i_{1}, i_{2}, \ldots, i_{d}}=\left\{v_{j_{1}}^{i_{1}}, v_{j_{2}}^{i_{2}}, \ldots, v_{j_{d}}^{i_{d}}\right\}$. We put $f_{i_{1}, i_{2}, \ldots, i_{d}}$ in the set $\mathcal{F}^{\prime}$.

5. For clarity of arguments in what follows, we give some more definitions. For each $f=\left\{v_{j_{1}}, v_{j_{2}}, \ldots, v_{j_{d}}\right\} \in \mathcal{F}$, the subfamily of hyperedges $\mathcal{F}_{v_{j_{1}}, v_{j_{2}}, \ldots, v_{j_{d}}}=$ $\left.\left\{f_{i_{1}, i_{2}, \ldots, i_{d}}\right\}=\left\{v_{j_{1}}^{i_{1}}, v_{j_{2}}^{i_{2}}, \ldots, v_{j_{d}}^{i_{d}}\right\} \mid i_{1}, i_{2}, \ldots, i_{d} \in\{1, \ldots, d\}\right\}$ is called a subsystem of $\left\{v_{j_{1}}, v_{j_{2}}, \ldots, v_{j_{d}}\right\}$. Also, $\mathcal{F}_{v_{j_{1}}, v_{j_{2}}, \ldots, v_{j_{d}}}$ is called a subsystem of $f$, for all hyperedges $f \in \mathcal{F}_{v_{j_{1}}, v_{j_{2}}, \ldots, v_{j_{d}}}$. It follows from the previous definition that a row in a subsystem corresponds to the $d$ copies of a vertex participating in the subsystem. 
Claim $1(U, \mathcal{F}, k)$ is a Yes instance of $d$-Hitting SET if and only if $\left(U^{\prime}, \mathcal{F}, d k\right)$ is a YeS instance of $d$-Hitting SET.

Next, we give a reduction from the instance $\left(U^{\prime}, \mathcal{F}, d k\right)$ of $d$-Hitting Set to a instance of HyPERPLANE POINT HS. The correctness of this reduction shows that there is a polynomial time reduction from $d$-Hitting Set to Hyperplane POINT HS such that the parameter transformation is linear.

We construct the following instance of HyPERPLANE POINT HS:

1. Using Lemma 5 , we construct a set $\mathcal{P}$ of $d n$ points, same as the number of elements in the universe $U^{\prime}$. We arbitrarily assign each element of $U^{\prime}$ to a unique point in $\mathcal{P}$.

2. For a hyperedge $f \in \mathcal{F}^{\prime}$, let $H_{f}$ be the hyperplane defined by the $d$ points contained in $f$. The set $\mathcal{H}$ is the family of such hyperplanes.

Claim $2(U, \mathcal{F}, k)$ is a YES instance of $d$-Hitting SET if and only if $(\mathcal{H}, d k)$ is $a$ Yes instance of Hyperplane PoINT HS in $\mathbb{R}^{d}$.

To prove this claim, we need the following claim regarding a solution set with minimum number of points outside $\mathcal{P}$.

Claim 3 Let $Q$ be a minimum sized set of points that covers all the hyperplanes in $\mathcal{H}$. Also, assume that $Q$ has the minimum possible points in $Q \backslash \mathcal{P}$. Moreover, let $q \in Q \backslash \mathcal{P}$ that covers the minimum number of hyperedges uniquely. We assume that there is no other set $Q^{\prime}$ of the same size as $Q$, with $\left|Q^{\prime} \backslash \mathcal{P}\right|=|Q \backslash \mathcal{P}|$ and with a $q^{\prime} \in Q^{\prime} \backslash \mathcal{P}$ that covers strictly less number of hyperedges uniquely in $Q^{\prime}$ than $q$ does in $Q$. Then for any element $v \in U^{\prime} \backslash Q$, at most $d-1$ hyperedges containing $v$ can have no intersection with $Q$.

Proof. Firstly, by the condition of minimality on $Q$, each point in $Q \backslash \mathcal{P}$ must uniquely cover at least 2 hyperplanes in $\mathcal{H}$. Otherwise we could find a equal-sized solution $Q^{\prime}$ where $\left|Q^{\prime} \backslash \mathcal{P}\right|<|Q \backslash \mathcal{P}|$, which is a contradiction.

Suppose that there is a vertex $v \in U^{\prime} \backslash Q$ such that at least a family $\mathcal{H}^{\prime}$ of $d$ hyperedges in $\mathcal{H}$ containing $v$ can have no intersection with $Q$. These $d$ hyperplanes are covered by a set $Q^{\prime}$ of points that are in $Q \backslash \mathcal{P}$. Suppose $Q^{\prime}=\left\{u_{1}, \ldots, u_{\ell}\right\}$ such that the for each $j \in\{1, \ldots, \ell\}, u_{j}$ uniquely covers $c_{j}$ hyperplanes of $\mathcal{H}^{\prime}$. By definition, $\Sigma_{j} c_{j}=d$. By the minimality condition of $Q$ and property (2) of Lemma 5 , each such point in $Q^{\prime} \subseteq Q \backslash \mathcal{P}$ uniquely covers between 2 to $d$ hyperplanes of $\mathcal{H}$. Thus, for each $j \in\{1,2, \ldots, \ell\}$ the vertex $u_{j}$ covers at most $d-c_{j}$ hyperplanes not in $\mathcal{H}^{\prime}$. We call the family of all hyperplanes covered by vertices of $Q^{\prime}$ as $\mathcal{H}^{\prime \prime}$. This family has at most $d(d-1)+d$ hyperplanes. We construct the following set $\hat{Q}$ :

- All points of $Q \backslash Q^{\prime}$ are included in $\hat{Q}$. The point $v$ is also included.

- For each $j \in\{1, \ldots, \ell\}$, let $\mathcal{H}_{j}$ be the subfamily of at most $d-c_{j}$ hyperplanes that are uniquely covered by the vertex $u_{j}$ and which are not in $\mathcal{H}^{\prime}$. Starting from $j=1$, we build a subfamily $\mathcal{H}_{j}^{\prime}$ and find a point $u_{j}^{\prime}$ corresponding to $u_{j}$. First all the hyperplanes in $\mathcal{H}_{j}$ are added to $\mathcal{H}_{j}^{\prime}$. Then, iterating a variable $t$ 
from $j+1$ to $\ell$, we add the hyperplanes in $\mathcal{H}_{t}$ till there are $d$ hyperplanes or all hyperplanes in $\bigcup_{t>j} \mathcal{H}_{t}$ have been added. Take a point in the intersection of $\mathcal{H}_{j}^{\prime}$ and name that point $u_{j}^{\prime}$. We show that the last nonempty subfamily $\mathcal{H}_{t}$ must be for $t<\ell$. Suppose not. Then by definition, when we consider the last point $u_{\ell}$, the number of hyperplanes in $\mathcal{H}_{\ell}$ that are not yet covered by $\left\{u_{1}^{\prime}, \ldots, u_{\ell-1}^{\prime}\right\}$ are at most $d-c_{\ell}-\Sigma_{j<\ell} c_{j}=d-\Sigma_{j \leq \ell} c_{j}=0$. Therefore, $\left\{c_{1}^{\prime}, c_{2}^{\prime}, \ldots, c_{\ell-1^{\prime}}\right\}$ cover all the hyperplanes in $\mathcal{H}^{\prime \prime} \backslash \mathcal{H}^{\prime}$. By definition of $\mathcal{H}^{\prime}$, the set $\left\{v, c_{1}^{\prime}, c_{2}^{\prime}, \ldots, c_{\ell-1^{\prime}}\right\}$ covers all the hyperplanes in $\mathcal{H}^{\prime \prime}$.

By definition the size of $\hat{Q}$ is at most that of $Q$. However, the number of vertices in $\hat{Q} \backslash \mathcal{P}$ is strictly less than the number of vertices in $Q \backslash \mathcal{P}$. This is a contradiction to the definition of $Q$.

Hence, we have proven the claim.

The proofs of the other claims can be found in the full version of the paper. Due to Claim 2, we show that there is a linear parameter transformation from $d$ Hitting Set to Hyperplane Point HS in $\mathbb{R}^{d}$. This implies that Hyperplane POINT HS in $\mathbb{R}^{d}$ cannot have a kernel of size $\mathcal{O}\left(k^{d-\epsilon}\right)$ if co-NP $\nsubseteq \mathrm{NP} /$ poly.

The following Corollary follows from Theorem 6 and Lemma 4 .

Corollary 7. Point Hyperplane Cover in $\mathbb{R}^{d}$ has a kernel of size $\Theta\left(k^{d}\right)$ if co-NP $\nsubseteq \mathrm{NP} /$ poly.

Using similar techniques, we also obtain tight kernels for Projective Point Hyperplane COVER in $\mathbb{R}^{d}$.

Lemma 8. Projective Point Hyperplane Cover in $\mathbb{R}^{d}$ has a kernel of size $\Theta\left(k^{d-1}\right)$ if co-NP $\nsubseteq \mathrm{NP} /$ poly.

By the method suggested by Dell an Melkebeek [8], we can show a lower bound on the number of points of a polynomial kernel for PoINT HyPERPLANE COver in $\mathbb{R}^{d}$, for each fixed positive integer $d$.

Lemma 9. Point Hyperplane Cover in $\mathbb{R}^{d}$ cannot have a kernel with $\mathcal{O}\left(k^{d-\epsilon}\right)$ points if co-NP $\nsubseteq \mathrm{NP} /$ poly.

Since Point Hyperplane Cover and Hyperplane Point HS are equivalent problems, we obtain the following corollary.

Corollary 10. HyPerPlane PoINT HS in $\mathbb{R}^{d}$ cannot have a kernel with $\mathcal{O}\left(k^{d-\epsilon}\right)$ hyperplanes if co-NP $\nsubseteq \mathrm{NP} /$ poly.

We also obtain the following corollary.

Corollary 11. Projective Point Hyperplane Cover in $\mathbb{R}^{d}$ cannot have a kernel with $\mathcal{O}\left(k^{d-1-\epsilon}\right)$ points if co-NP $\nsubseteq \mathrm{NP} /$ poly. 


\section{Covering Polynomials of bounded degree with Points}

In this section, we consider the $D$-Polynomial Point HS problem and show that this problem is equivalent to HYPERPLANe POINT HS in a higher dimensional space. We utilize this to give tight polynomial kernels for $D$-PoLYNOMial PoINT HS, when the underlying vector space of polynomials is $\alpha$-good.

Recall that in $D$-Polynomial Point HS, a vector space $\mathcal{R}$ of $D$-degree polynomials in Poly $_{D}\left[X_{1}, X_{2}, \ldots, X_{d}\right]$ is specified. The input is a set $\mathcal{F}$ of $n$ polynomials from $\mathcal{R}$ and the objective is to find at most $k$ points in $\mathbb{R}^{d}$ that cover all the input polynomials.

We utilize the Veronese mapping from a vector space of $D$-degree polynomials to the subsystem of hyperplanes in Euclidean space $\mathbb{R}^{b}$. Such a mapping is a bijective mapping between the vector space of $D$-degree polynomials and the hyperplanes in $\mathbb{R}^{b}$. However, the mapping need not be an onto mapping from $\mathbb{R}^{d}$ to $\mathbb{R}^{b}$. Let $\operatorname{Ver}_{\mathcal{R}}\left(\mathbb{R}^{d}\right)$ be the image of $\mathbb{R}^{d}$ under the Veronese mapping $\Phi_{\mathcal{R}}$. Thus, $\operatorname{Ver}_{\mathcal{R}}\left(\mathbb{R}^{d}\right) \subseteq \mathbb{R}^{b}$. We show that the HyPERPLANE POINT HS problem for an $\alpha$-good vector space $\mathcal{R}$ in $\mathbb{R}^{b}$ when the solution set is restricted to belonging to $\operatorname{Ver}_{\mathcal{R}}\left(\mathbb{R}^{d}\right)$, does not have a $\mathcal{O}\left(k^{b-\epsilon}\right)$ kernel unless co-NP $\subseteq \mathrm{NP} /$ poly.

Before this, we require a few results regarding the behaviour of points under the Veronese mapping.

First, we show that a set of $n$ points in $\mathbb{R}^{d}$ that are in general position with respect to $\mathcal{R}$ are mapped to a set of $n$ points in $\mathbb{R}^{b}$ in general position with respect to hyperplanes in $\mathbb{R}^{b}$.

Claim 4 Let $\mathcal{P}$ be a set of points in $\mathbb{R}^{d}$, and $\mathcal{R}$ be a subspace of $\operatorname{Poly}_{D}\left[X_{1}, \ldots, X_{d}\right]$ with a basis $\left\{f_{1}(X), \ldots, f_{b}(X), 1\right\}$ where $X=\left(X_{1}, \ldots, X_{d}\right)$.

1. If the set $\mathcal{P}$ is in general position with respect to the polynomial family $\mathcal{R}$ then the image $\Phi_{\mathcal{R}}(\mathcal{P})$, under the Veronese mapping $\Phi_{\mathcal{R}}$, is a $|\mathcal{P}|$-sized set in general position with respect to hyperplanes in $\mathbb{R}^{b}$.

2. Let $S=\left\{q_{1}, \ldots, q_{\ell}\right\} \subseteq \Phi_{\mathcal{R}}(\mathcal{P})$ be in general position with respect to hyperplanes in $\mathbb{R}^{b}$. Then the set $S^{\prime}=\left\{p_{1}, \ldots, p_{\ell}\right\}$, where $p_{i} \in \Phi_{\mathcal{R}}^{-1}\left(q_{i}\right) \cap \mathcal{P}$, will be a $|S|$-sized set in general position with respect to $\mathcal{R}$.

Proof. 1. First, observe that if the map $\Phi_{\mathcal{R}}$ is injective on $\mathcal{P}$ then the result will directly follow. However, in general, the map $\Phi_{\mathcal{R}}$ need not be an injective mapping on an arbitrary set of $n$ points in $\mathbb{R}^{d}$. We show that $\Phi_{\mathcal{R}}$ is injective when restricted to $\mathcal{P}$ if $\mathcal{P}$ is in general position with respect to $\mathcal{R}$. To reach a contradiction, let $\Phi_{\mathcal{R}}\left(p_{1}\right)=\Phi_{\mathcal{R}}\left(p_{2}\right)$ where $p_{1}, p_{2}\left(\neq p_{1}\right) \in \mathcal{P}$. Let $S \subseteq \mathcal{P}$ be of size $b+1$ and $p_{1}, p_{2} \in \mathcal{P}$. Observe that the set $\Phi_{\mathcal{R}}(S)$ will have less than $b+1$ points and this will imply that there exists a hyperplane $\sum_{i=1}^{b} \lambda_{i} Z_{i}+\lambda_{b+1}=0$ on which the set $\Phi_{\mathcal{R}}(S)$ will lie. But this implies that the polynomial $\sum_{i=1}^{b} \lambda_{i} f_{i}(X)+\lambda_{b+1}=0$ will be satisfied by all the points in $S$. Thus, we have reached a contradiction from the fact that the point set $\mathcal{P}$ was in general position.

2. The second part of the Claim follows directly from the construction of the mapping $\Phi_{\mathcal{R}}$. 
Next, for each $n$ we construct a set of $n$ points that satisfy the conditions of Lemma 5 and belong to $\operatorname{Ver}_{\mathcal{R}}\left(\mathbb{R}^{d}\right)$, where $\mathcal{R}$ is $\alpha$-good. This construction is mainly done in $\mathbb{R}^{d}$ and follows exactly along the lines of the proof of Lemma 5 .

Lemma 12. Let $\mathcal{R}$ be a $\alpha$-good vector space of $D$-degree polynomials in $\mathbb{R}^{d}$ and let the Veronese mapping $\Phi_{\mathcal{R}}$ linearize $\mathcal{R}$ into $\mathbb{R}^{b}$. Let $\operatorname{Ver}_{\mathcal{R}}\left(\mathbb{R}^{d}\right)$ be the image of $\Phi_{\mathcal{R}}$. Then for every $n \in \mathbb{Z}^{+}$, there is a poly $(n)$ time algorithm to construct a set $\mathcal{P}$ of $n$ points in $\mathbb{R}^{b}$ that have the following properties:

(1) The points are in general position with respect to hyperplanes in $\mathbb{R}^{b}$.

(2) Let $\mathcal{H}$ be the family of hyperplanes defined by each set of $b$ points from $\mathcal{P}$. The hyperplanes in the family $\mathcal{H}$ are in general position, i.e., given $r$ hyperplanes $H_{1}, \ldots, H_{r}$ in $\mathcal{H}$ with $r \leq b$ the dimension of the affine space $\cap_{i=1}^{r} H_{r}$ is $b-r$.

(3) For any point $p$ in $\mathbb{R}^{b} \backslash \mathcal{P}$, there are at most b hyperplanes in $\mathcal{H}$ that contain $p$.

Proof. As in the proof of Lemma 5, we will construct the set $\mathcal{P}$ inductively. We start with a set $\mathcal{P}_{b}$ of size $b$ such that the set is in general position with respect to $\mathcal{R}$. This can be constructed in $O(1)$ time as $\mathcal{R}$ is $\alpha$-good. We then extend this set one point at a time using points from the grid (as in the proof of Lemma 5). Assume that for $b \leq t<n$, we have constructed a point set $\mathcal{P}_{t}$ that satisfies the above conditions. The points forbidden to be added to the set $\mathcal{P}_{t}$ will lie on a bounded number of polynomials from $\mathcal{R}$ and we will call these polynomials forbidden polynomials. The hyperplane that is in bijective correspondence with a forbidden polynomial under the Veronese mapping $\Phi_{\mathcal{R}}$ is called a forbidden hyperplane. As in the proof of Lemma 4, we can show, using the Veronese mapping $\Phi_{\mathcal{R}}$, that the number of forbidden hyperplanes arising due to conditions (1), (2) and (3) is bounded by $O\left(t^{b}\right), O\left(t^{b^{2}+b-1}\right)$ and $O\left(t^{b^{2}+b-1}\right)$ respectively. This also gives a bound on the number of forbidden polynomials.

As we have an upper bound on the number of forbidden polynomials, we can now use the same trick to generate points satisfying conditions (1) to (3) as Lemma 4. In this case we take a $d$-dimensional $m \times \cdots \times m$ grid with $m=n^{b^{2}+b}$ and use the fact that given any polynomial from $\mathcal{R}$, the number of points of the grid hitting it is bounded by $m^{d-\alpha}$. This completes the proof.

This helps us to prove a kernel lower bound on the restricted version of Hyperplane Point Cover described above.

Lemma 13. Let $\mathcal{R}$ be an $\alpha$-good vector space of $D$-degree polynomials in $\mathbb{R}^{d}$ and let the Veronese mapping $\Phi_{\mathcal{R}}$ linearize $\mathcal{R}$ into $\mathbb{R}^{b}$. Then HyPERPlane Point COVER, when the solution is restricted to belong to $\operatorname{Ver}_{\mathcal{R}}\left(\mathbb{R}^{d}\right)=\Phi_{\mathcal{R}}\left(\mathbb{R}^{d}\right)$, cannot have a kernel of size $\mathcal{O}\left(k^{b-\epsilon}\right)$ unless co-NP $\subseteq \mathrm{NP} /$ poly.

Proof. The construction of $n$ points in $\operatorname{Ver}_{\mathcal{R}}\left(\mathbb{R}^{d}\right)$ described in Lemma 12 has all the properties described in Lemma 5 . The rest of the proof follows exactly as the proof of Theorem 6 . 
Finally, the following Theorem is derived from Lemma 13 by utilizing the Veronese mapping $\Phi_{\mathcal{R}}$.

Theorem 14. $D$-Polynomial Point HS for an $\alpha$-good vector space $\mathcal{R}$ in $\mathbb{R}^{d}$, and having the Veronese mapping into $\mathbb{R}^{b}$, (i) has a polynomial kernel of size $\mathcal{O}\left(k^{b}\right)$, (ii) does not have a polynomial kernel of size $\mathcal{O}\left(k^{b-\epsilon}\right)$, unless co-NP $\subseteq \mathrm{NP} /$ poly.

Proof. To prove the tightness of a $\mathcal{O}\left(k^{b}\right)$ kernel for $D$-Polynomial Point HS in $\mathbb{R}^{d}$ with the Veronese mapping into $\mathbb{R}^{b}$, first we prove (i) by giving an upper bound on the size of a kernel. Let the polynomials in an instance of $D$-Polynomial Point HS come from the vector space $\mathcal{R}$, as defined earlier. The Veronese mapping $\Phi_{\mathcal{R}}$ is a reduction from $D$-Polynomial Point HS in $\mathbb{R}^{d}$ to Hyperplane Point HS in $\mathbb{R}^{b}$. Thus, since Hyperplane Point HS in $\mathbb{R}^{b}$ has a $\mathcal{O}\left(k^{b}\right)$ kernel [17], so does $D$-Polynomial Point HS in $\mathbb{R}^{d}$ with the Veronese mapping into $\mathbb{R}^{b}$.

To prove (ii), we use the Veronese mapping on the vector space $\mathcal{R}$ of $D$-degree polynomials more carefully. Let the hyperplanes, to which the polynomials are mapped, be in $\mathbb{R}^{b}$. The mapping is a bijective function. Thus, in order to obtain the required result, we give a reduction from Hyperplane Point HS in $\mathbb{R}^{b}$, where the solution set of points come from $\operatorname{Ver}_{\mathcal{R}}\left(\mathbb{R}^{d}\right)$. The reduction is simply the reverse function of the Veronese mapping. Suppose an instance $(\mathcal{H}, k)$ of Hyperplane Point HS where the solution set of points belong to $\operatorname{Ver}_{\mathcal{R}}\left(\mathbb{R}^{d}\right)$ reduces to the instance $\left(\mathcal{H}^{\prime}, k\right)$ of $D$-Polynomial Point HS. If $(\mathcal{H}, k)$ is a YeS instance, then there is a set $S$ of at most $k$ points in $\mathbb{R}^{b}$ that covers all the hyperplanes in $\mathcal{H}$. Consider the set $S^{\prime}$ of points in $\mathbb{R}^{d}$ by taking one preimage of each point in $S$. The set $S^{\prime}$ is exactly the same size as $S$, and therefore contains at most $k$ points. Moreover, by definition of the Veronese mapping, $S^{\prime}$ covers all the polynomials in $\mathcal{H}^{\prime}$. Therefore, $\left(\mathcal{H}^{\prime}, k\right)$ is also a Yes instance for $D$-Polynomial POINT HS.

On the other hand, if $\left(\mathcal{H}^{\prime}, k\right)$ is a Yes instance of $D$-Polynomial Point HS, then there is a set $S^{\prime}$ of at most $k$ points that cover all the polynomials in $\mathcal{H}^{\prime}$. The image of $S^{\prime}$ under the Veronese mapping will be of size at most $S^{\prime}$ and will cover the family $\mathcal{H}$. Therefore, $(\mathcal{H}, k)$ will be a Yes instance of Hyperplane Point Cover when the solution points can come only from $\operatorname{Ver}_{\mathcal{R}}\left(\mathbb{R}^{d}\right)$. Thus, by Lemma 13 , we conclude that $D$-POLYNomial Point HS cannot have a kernel of size $\mathcal{O}\left(k^{b-\epsilon}\right)$ unless co-NP $\subseteq \mathrm{NP} /$ poly.

\section{Open Problems}

The $D$-Point Polynomial Cover problem in $\mathbb{R}^{d}$ requires a set of $n$ points in $\mathbb{R}^{d}$ to be covered by at most $k D$-degree polynomials. Although polynomial kernels for $D$-Point Polynomial Cover in $\mathbb{R}^{d}$ can be exhibited, tight lower bounds for this problem are unknown. 


\section{References}

1. P. Afshani, E. Berglin, I. van Duijn, and J. S. Nielsen. Applications of Incidence Bounds in Point Covering Problems. In SoCG, pages 60:1-60:15, 2016.

2. P. Alimonti and V. Kann. Some APX-completeness results for cubic graphs. TCS, 237(1-2):123-134, 2000.

3. P. Ashok, S. Kolay, N. Misra, and S. Saurabh. Unique covering problems with geometric sets. In COCOON, pages 548-558. Springer, 2015.

4. P. Ashok, S. Kolay, and S. Saurabh. Multivariate complexity analysis of geometric red blue set cover. Algorithmica, pages 1-31, 2015.

5. J. Boissonnat, K. Dutta, A. Ghosh, and S. Kolay. Kernelization of the subset general position problem in geometry. In MFCS, pages 25:1-25:13, 2017.

6. K. Bringmann, L. Kozma, S. Moran, and N. S. Narayanaswamy. Hitting set for hypergraphs of low VC-dimension. In $E S A$, pages 23:1-23:18, 2016.

7. M. Cygan, F. V. Fomin, E. Kowalik, D. Lokshtanov, D. Marx, M. Pilipczuk, M. Pilipczuk, and S. Saurabh. Parameterized Algorithms, volume 3. Springer, 2015.

8. H. Dell and D. Van Melkebeek. Satisfiability allows no nontrivial sparsification unless the polynomial-time hierarchy collapses. In STOC, pages 251-260. ACM, 2010.

9. I. Dinur and D. Steurer. Analytical approach to parallel repetition. In $S T O C$, pages 624-633. ACM, 2014.

10. M. Dom, M. R. Fellows, and F. A. Rosamond. Parameterized complexity of stabbing rectangles and squares in the plane. In WALCOM, pages 298-309. Springer, 2009.

11. R. G. Downey and M. R. Fellows. Parameterized Complexity. Springer-Verlag, 1999. 530 pp.

12. J. Flum and M. Grohe. Parameterized Complexity Theory (Texts in Theoretical Computer Science. An EATCS Series). Springer-Verlag New York, Inc., Secaucus, NJ, USA, 2006.

13. P. Giannopoulos, C. Knauer, and S. Whitesides. Parameterized complexity of geometric problems. The Computer Journal, 51(3):372-384, 2008.

14. P. Heggernes, D. Kratsch, D. Lokshtanov, V. Raman, and S. Saurabh. Fixedparameter algorithms for cochromatic number and disjoint rectangle stabbing via iterative localization. Inf. Comput., 231:109-116, 2013.

15. R. M. Karp. Reducibility among combinatorial problems. In 50 Years of Integer Programming 1958-2008 - From the Early Years to the State-of-the-Art, pages 219-241. 2010.

16. S. Kratsch, G. Philip, and S. Ray. Point line cover: the easy kernel is essentially tight. TALG, 12(3):40, 2016.

17. S. Langerman and P. Morin. Covering things with things. DCG, 33(4):717-729, 2005.

18. J. Matoušek. Lectures on Discrete Geometry, volume 212. Springer Science \& Business Media, 2002.

19. N. Megiddo and A. Tamir. On the complexity of locating linear facilities in the plane. Oper. Res. Lett., 1(5):194-197, Nov. 1982.

20. V. V. Vazirani. Approximation Algorithms. Springer Science \& Business Media, 2013. 